EPJ Web of Conferences 19, 04006 (2012)

DOI: $10.1051 /$ epjconf/20121904006

(C) Owned by the authors, published by EDP Sciences, 2012

\title{
Gauging the Galactic thick disk with RR Lyrae stars
}

\author{
C. Mateu ${ }^{1,2, a}$, K. Vivas ${ }^{1}$, J.J. Downes ${ }^{1,2}$, C. Briceño ${ }^{1}$ and G. Cruz ${ }^{3}$ \\ ${ }^{1}$ Centro de Investigaciones de Astronomía (CIDA), La Hechicera, Apartado Postal 264, \\ Mérida 5101-A, Venezuela \\ ${ }^{2}$ Facultad de Ciencias, Universidad Central de Venezuela, Caracas, Venezuela \\ ${ }^{3}$ Centro de Astrobiología, 28850 Torrejón de Ardoz, Madrid Spain
}

\begin{abstract}
In this contribution we present results from the QUEST RR Lyrae Survey of the thick disk. The survey spans $\sim 480$ sq. deg. at low latitude $|b|<30^{\circ}$, with multi-epoch VRI observations, obtained with the QUEST-I camera at the $1 \mathrm{~m}$ Jürgen Stock Schmidt telescope located at the National Astronomical Observatory of Venezuela. This constitutes the first deep RR Lyrae survey of the Galactic thick disk conducted at low galactic latitudes, covering simultaneously a large range in radial $(8<R(k p c)<60)$ and perpendicular $(0<|z|(k p c)<20)$ distance from the Galactic Plane. The spatial coverage of the survey together with the multi-band multi-epoch photometry allowed for the derivation of the thick disk structural parameters from in situ RR Lyrae stars having accurate distances (errors $<7 \%$ ) and individual reddenings derived from each star's color curve at minimum light. Moreover, the use of RR Lyrae stars as tracers ensures negligible contamination from the Galactic thin disk. We find a thick disk mean scale height $h_{Z}=0.94 \pm 0.11 \mathrm{kpc}$ and scale length $h_{R}=3.2 \pm 0.4 \mathrm{kpc}$, derived from the vertical and radial mean density profiles of RR Lyrae stars. We also find evidence of thick disk flaring and results that may suggest the thick disk radial density profile shows signs of antitruncation. We discuss our findings in the context of recent thick disk formation models.
\end{abstract}

\section{THE QUEST-I RR LYRAE SURVEY OF THE THICK DISK}

The goal of our work was to characterize the structure of the Milky Way thick disk using RR Lyrae stars as tracers, by means of a large-scale survey at low galactic latitudes. The present survey spans an area of $\sim 480$ sq. deg. in the galactic latitud range $-30^{\circ} \leqslant b \leqslant+25^{\circ}$ approximately towards the Galactic anticenter $190^{\circ}<l<230^{\circ}$, with multi-epoch VRI observations for $6.5 \times 10^{6}$ objects, obtained between 1998 and 2008 with the QUEST-I camera at the 1m Jürgen Stock Schmidt telescope located at the National Astronomical Observatory of Venezuela.

We identified $160 \mathrm{RR} a b$ and $51 \mathrm{RR} c$ stars in the magnitude range $14<V<18.5$, with a completeness estimated in $95 \%$ and $80 \%$ respectively. Distances to RR $a b$ stars were derived using individual reddenings computed for each star from its mean $V-R$ and $V-I$ color curves during the minimum light phase $[1,2]$, resulting in typical distance errors of $\sim 7 \%$. The present therefore constitutes the first deep RR Lyrae survey of the Galactic thick disk conducted at low galactic latitudes, covering simultaneously a large range in radial $(8<R(k p c)<60)$ and perpendicular $(0<|z|(k p c)<20)$ distance from the Galactic Plane. The survey coverage is illustrated in Figure 1, in comparison with previous tracer surveys of the Galactic thick disk ([3-6]).

\footnotetext{
ae-mail: cmateu@cida.ve
}

This is an Open Access article distributed under the terms of the Creative Commons Attribution-Noncommercial License 3.0, which permits unrestricted use, distribution, and reproduction in any noncommercial medium, provided the original work is properly cited. 


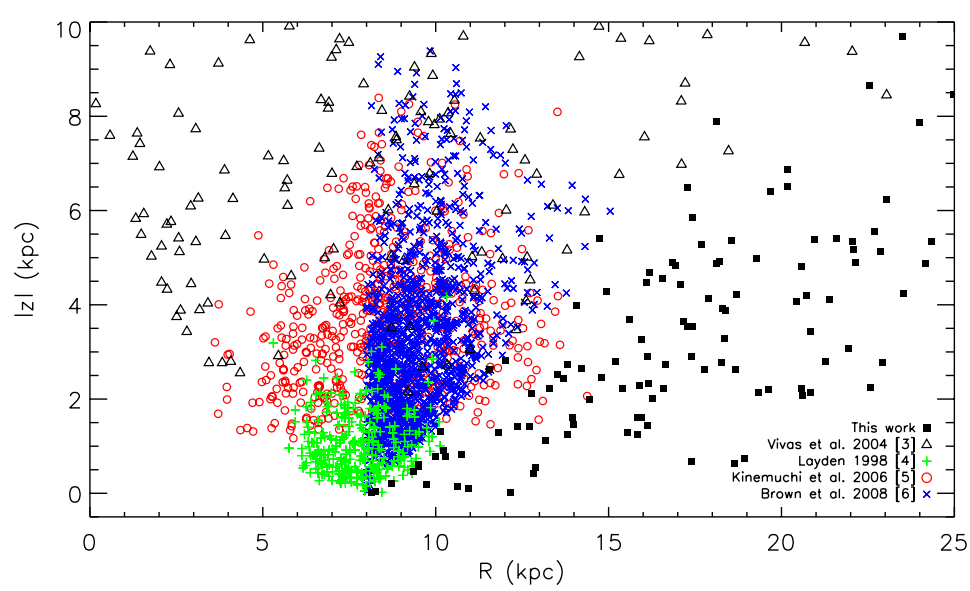

Figure 1. Survey coverage in cylindric galactocentric coordinates $|z|$ versus $R$, compared to previous thick disk tracer surveys. RRLS from [3] were included to supplement the survey at high latitudes.

\section{THE GALACTIC THICK DISK TRACED WITH RR LYRAE STARS}

We computed cumulative mean densities $\rho_{R R}(\leq R)$ and $\rho_{R R}(\leq z)$ of RRLS (only for type $a b$ RRLS) in the survey volume up to a projected galactocentric radius $R$ and distance perpendicular to the Galactic Plane $|z|$ respectively. We derived Halo density profile parameters by fitting the observed profiles for RRLs outside the Galactic Plane $(|z| \geq 6 \mathrm{kpc})$. We used these to account for the contribution of Halo RRLS near the Galactic Plane $(|z|<6 \mathrm{kpc})$, where we fitted the observed radial and vertical profiles to derive the thick disk parameters. We obtained a mean scale height of $h_{Z}=(0.94 \pm 0.11) \mathrm{kpc}$ and mean scale length $h_{R}=(3.2 \pm 0.4) \mathrm{kpc}$ for the thick disk.

We also find that in order to appropiately fit the full radial density profile, the scale length $h_{R}$ must be shorter for $R \lesssim 11.5 \mathrm{kpc}$ than for longer distances, suggesting the thick disk might have an antitruncated or Type III profile, similar to those observed in external galaxy disks [7]. Finally, we also fitted the vertical density profile of RRLs in five different radial distance intervals, in order to explore the behaviour of the scale length as a function of $R$. The scale height is found to increase at a rate $\Delta h_{Z} / h_{Z}=0.9$, i.e. $90 \%$ if $R$ increases in one scale length. Our results for the mean scale height and scale length are consistent with current predictions of thick disk formation models by thin disk heating [8] and gas-rich mergers [9]; however, our results for the observed flare amplitude favour current gasrich merger models [10].

\section{References}

[1] Guldenschuh, K. A. et al. 2005, PASP, 117, 721

[2] Kunder, A., Chaboyer, B., \& Layden, A. 2010, AJ, 139,415

[3] Vivas, A. K. et al. 2004, AJ, 127, 1158

[4] Layden, A. 1998, AJ, 115, 193

[5] Kinemuchi, K., Smith, H. A., Woźniak, P. R. \& McKay, T. A., 2006, AJ, 132, 1202

[6] Brown, W. R. et al., 2008, AJ, 135, 564

[7] Pohlen, M., Balcells, M., Lütticke, R., \& Dettmar, R.-J. 2004, A\&A, 422, 465

[8] Villalobos, Á., \& Helmi, A., 2008, MNRAS, 391, 1806

[9] Brook, C. B., Kawata, D., Gibson, B. K., \& Freeman, K. C., 2004, ApJ, 612, 894

[10] Bournaud, F., Elmegreen, B. G., \& Martig, M., 2009, ApJ, 707, L1 